\title{
Severe bleeding from 'diversion pouchitis'
}

\author{
Christopher Heughan, MB, BChIr, FrCSC, James Barrowman, MB, PhD, FrCPC, \\ WILLIAM G POLLETT, MD, FRCSC
}

\begin{abstract}
A patient suffered severe bleeding from a defunctioned ileal pouch. Although this has not been described before, it may become more prevalent as more patients have ileal pouches to preserve anal function. The possible causes and a possible way of avoiding this catastrophe in the future are discussed. Can J Gastroenterol 1990;4(8):495-496
\end{abstract}

Key Words: Bleeding, Ileal pouch

\section{Hémorragie grave provenant d'une 'pouchite de détournement'}

RESUME: Un patient a souffert d'une hémorragie grave résultant d'un réservoir iléal "désaffecté". Bien que le phénomène n'ait pas encore été décrit, il se peut qu'il se produise plus souvent avec le nombre grandissant de patients ayant des réservoirs iléaux afin de conserver la fonction anale. Le présent article examine les causes possibles de cette catastrophe et une façon de l'éviter à l'avenir.

A 35-YEAR-OLD MAN WITH PROVEN ulcerative colitis underwent a subtotal colectomy and ileostomy for a toxic megacolon in August 1987.

In November 1988, he underwent mucosal proctectomy with construction of an ileal ' $S$ ' pouch and a tem- porary loop ileostomy. Recovery was uncomplicated apart from a fever and some drainage per rectum which settled on antibiotics. The patient was readmitted and his ileostomy closed in June 1989. He was readmitted 10 days later because of severe diarrhea. This settled

Health Sciences Centre, St John's, Newfoundland

Correspondence and reprints: Dr Christopher Heughan, Department of Surgery, Health

Sciences Centre, St John's, Newfoundland AIB 3V6. Telephone (709) 737 -6558

Received for publication June 13, 1990. Accepted June 25, 1990

rapidly on loperamide (Imodium; Janssen Pharmaceutica) and an ultrasound showed no evidence of pelvic abscess.

Three months later, in September 1989, he was again admitted because of weight loss, diarrhea and poor anal control. On this occasion, examination revealed a stricture at the site of the ileoanal anastomosis together with an area of disruption of the anastomosis. An anoplasty was performed but the diarrhea, incontinence and anal excoriation continued despite metronidazole and antidiarrheal agents.

Accordingly, a terminal ileostomy was performed in October 1989. The proximal end of the pouch was closed and left in the abdomen with a view to re-establishing the ileal pouch after the anastomosis had healed.

The patient recovered uneventfully and was discharged home in October 1989. He remained well until January 1990 when he was readmitted with severe bleeding per anum requiring seven units of fresh whole blood in the community hospital where the family 
physician had also applied pneumatic antishock trousers and placed a Foley catheter in his rectum to try, without success, to tamponade the bleeding.

After transfer, his pulse was 100 beats/min, blood pressure $100 / 60 \mathrm{mmHg}$, and hemoglobin $108 \mathrm{~g} / \mathrm{dL}$. There was a tender mass in the lower abdomen. A steady flow of blood was coming around the catheter in the anal canal.

A rigid sigmoidoscopy was attempted but the view was obscured by a mass of blood clot in the ileal pouch. The anal stricture would not permit passage of the instrument.

The patient underwent a laparotomy when a mass of clot in the pelvis was evacuated. The serosal surface of the ileal pouch appeared granular and was bleeding. The pouch was excised per abdomen leaving the anus intact. His ileostomy, which had receded, was revised.

The patient received a total of 13 units of packed red cells, two units of fresh frozen plasma and large volumes of crystalloid during the perioperative period. He made an uncomplicated recovery and was discharged home on the 10 th postoperative day.

\section{PATHOLOGY}

A review of the pathology of the original total colectomy confirmed acute inflammation confined to the mucosa and submucosa, consistent with ulcerative colitis. Aerobic and anaerobic cultures from the excised pouch taken during surgery were negative.

The excised pouch showed sub-

\section{REFERENCES}

1. Luukkonen P, Valtonen V, Sivontn A, Sipponen P, Jarvinen H. Fecal bacteriology and reservoir ileitis in patients operated on for ulcerative colitis. Dis Colon Rectum 1988;31:864-7.

2. Glotzer DJ, Glick ME, Goloman H. Proctitis and colitis following diversion of the fecal stream. Gastroenterology mucosal edema and nonspecific inflammatory change throughout the bowel wall and congestion and hemorrhage in the serosa.

\section{DISCUSSION}

There is, to the authors' knowledge, no previous description of severe bleeding from a defunctioned ileal pouch. The cause of the bleeding which occurred both into the lumen and from the serosal surface in this patient is not clear.

Examination of the excised pouch showed inflammation throughout the thickness of the ileal wall. However, the other features were not suggestive of Crohn's disease and a review of the original colonic pathology did not suggest anything other than ulcerative colitis.

Pouchitis is a well-recognized entity but its cause is obscure. Although there is a change in the luminal flora following conversion of ileum to a reservoir and although pouchitis responds to treatment with metronidazole, there appear to be no differences in flora between pouches of patients with the clinical syndrome of pouchitis and those without (1). Furthermore, no descriptions could be found in the literature of heavy bleeding caused by pouchitis.

Diversion colitis has been described as affecting defunctioned rectal stumps after Hartmann's operation (2). Although the histology is similar to that of ulcerative colitis (2-4), it does not respond to treatment with steroids (3).

1981;80:438-41

3. Korelitz Bl, Cheskin LJ, Sohn N, Sommers SC. The fate of the rectal segment after diversion of the fecal stream in Crohn's disease: Its implications for surgical management. J Clin Gastroenterol 1985;7:37-45.

4. Haas PA, Fox TA. The fate of the forgotten rectal pouch after Hartmann's
There is no change in flora which correlates with the clinical condition of diversion colitis which may occur after colectomy for tumour, diverticular disease or functional bowel disease (2) as well as for inflammatory bowel disease. Gross bleeding may occur from diversion colitis (5).

It has been suggested recently that diversion colitis may be due to a diver. sion of nutrients normally present in the fecal stream since the condition is improved by irrigation with short chain fatty acids (6). If this is so, it would be reasonable to speculate that a defunc. tioned ileal pouch may be similarly prone to acute infection caused by nor. mally harmless organisms (6) when deprived of adequate luminal nutrients.

Diversion ileitis has not been described in patients with a pouch who are awaiting routine closure of their ileostomy. This may be due to the relatively short time during which the pouch is not in the fecal stream. Alternatively, it may be that a loop ileostomy permits passage of enough material into the distal limb to keep the pouch in reasonable health.

Although this report is of an isolated case, it seems possible that, if the currently fashionable belief that a functioning anus is necessary for social and psychological well-being persists, more cases of life threatening bleeding may occur. If a pouch is out of the fecal stream for over three months, perhaps regular irrigation with nutrients such as short chain fatty acids might avert a possible catastrophe.

procedure without reconstruction. Am J Surg 1990;159:106-11.

5. Ona FV, Boger JN. Rectal bleeding due to diversion colitis. Am J Gastroenterol 1985;80:40-1.

6. Harig JM, Soergel KH, Komorowski RA, Wood CM. Treatment of diversion colitis with short-chain fatty acid irrigation. N Engl J Med 1989;320:23-8 


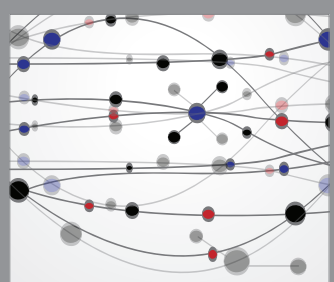

The Scientific World Journal
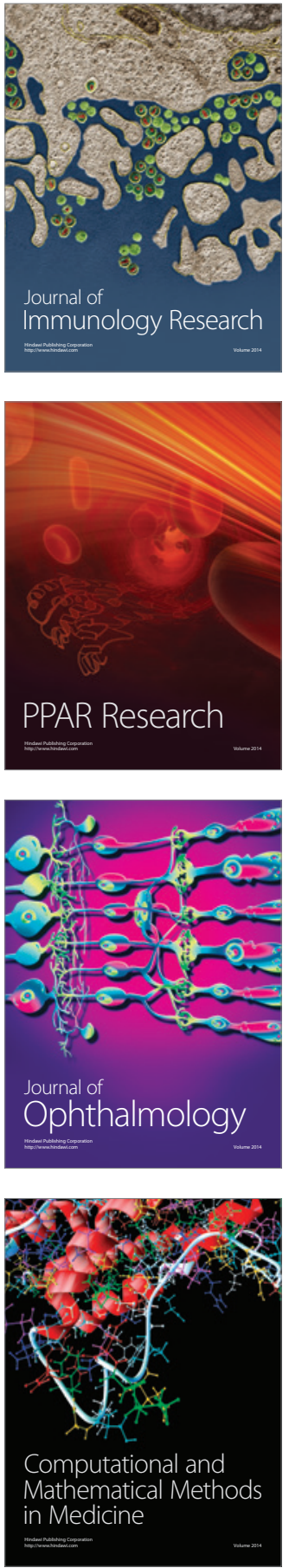

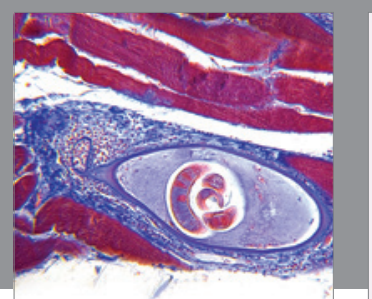

Gastroenterology Research and Practice

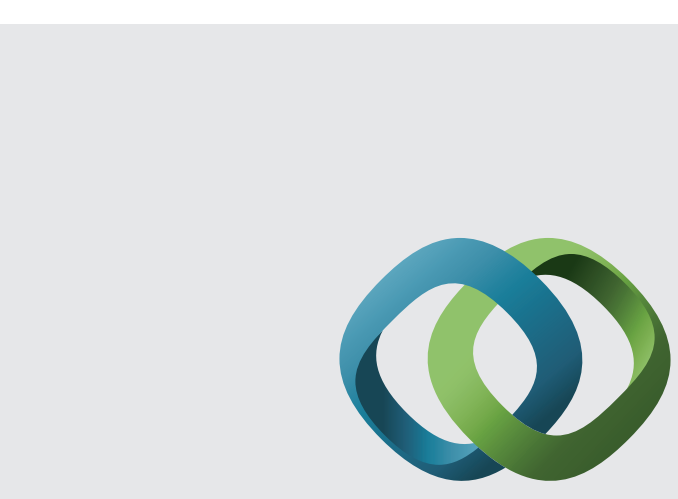

\section{Hindawi}

Submit your manuscripts at

http://www.hindawi.com
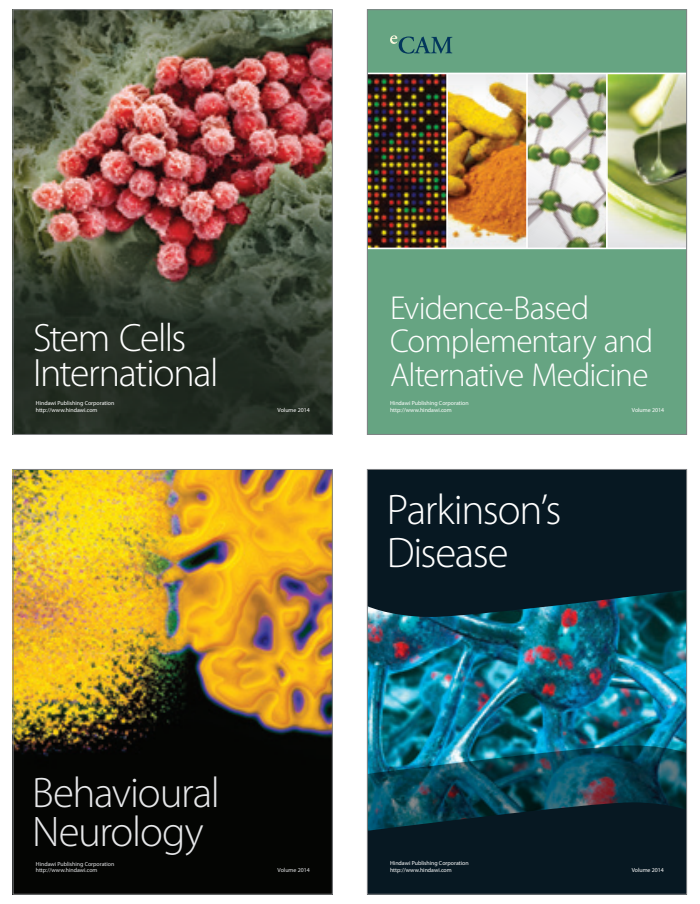
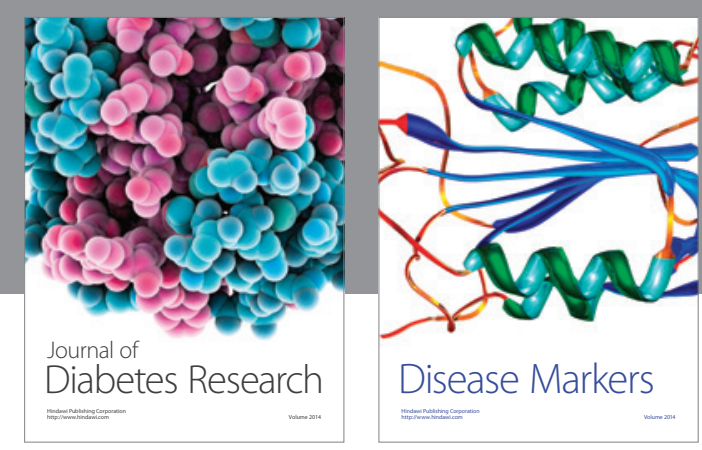

Disease Markers
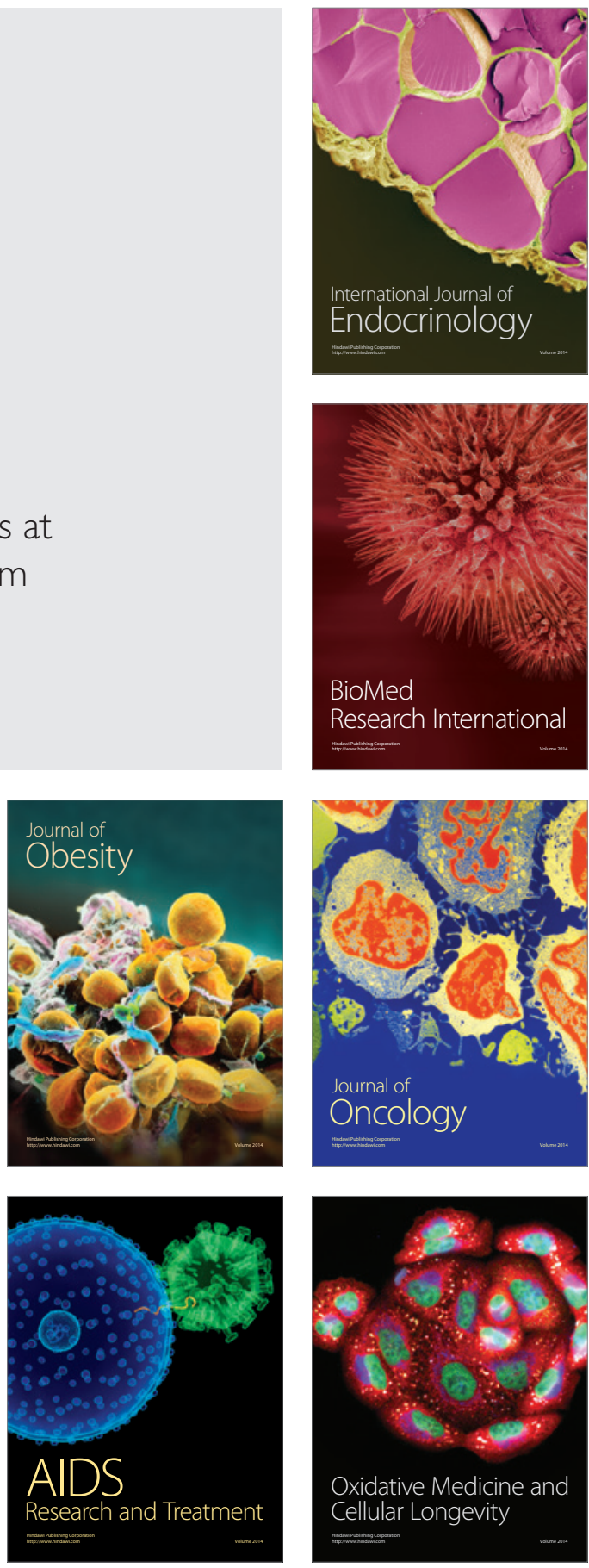